\title{
SOLVING THE TRUNCATED MOMENT PROBLEM SOLVES THE FULL MOMENT PROBLEM
}

\author{
JAN STOCHEL \\ Instytut Matematyki, Uniwersytet Jagielloński, ul. Reymonta 4, PL-30059 Kraków, Poland \\ e-mail: stochel@im.uj.edu.pl
}

(Received 17 November, 1999)

\begin{abstract}
It is shown that the truncated multidimensional moment problem is more general than the full multidimensional moment problem.

1991 Mathematics Subject Classification. Primary 44A60. Secondary 30E05.
\end{abstract}

Introduction. Denote by $\mathbb{Z}_{+}^{d}$ (resp. $\mathbb{C}^{d}$ ) the set of all $d$-tuples of nonnegative integers (respectively complex numbers). If $\alpha=\left(\alpha_{1}, \ldots, \alpha_{d}\right) \in \mathbb{Z}_{+}^{d}$ and $z=\left(z_{1}, \ldots, z_{d}\right) \in \mathbb{C}^{d}$, then we write $|\alpha|=\alpha_{1}+\ldots+\alpha_{d}, \quad z^{\alpha}=z_{1}^{\alpha_{1}} \cdots z_{d}^{\alpha_{d}} \quad$ and $\bar{z}=\left(\bar{z}_{1}, \ldots, \bar{z}_{d}\right)$. Let $F$ be a nonempty closed subset of $\mathbb{C}^{d}$ and let $c^{(n)}=\left\{c(\alpha, \beta): \alpha, \beta \in \mathbb{Z}_{+}^{d},|\alpha|+|\beta| \leq n\right\}$ be a finite multisequence of complex numbers $(n \geq 0)$. The truncated (multidimensional and complex) F-moment problem of order $n$ consists in determining conditions under which there exists a positive Borel measure $\mu$ on $\mathbb{C}^{d}$ such that the closed support supp $\mu$ of $\mu$ is contained in $F$ and $^{1}$

$$
c(\alpha, \beta)=\int z^{\alpha} \bar{z}^{\beta} d \mu(z), \quad \alpha, \beta \in \mathbb{Z}_{+}^{d},|\alpha|+|\beta| \leq n .
$$

A positive Borel measure $\mu$ on $\mathbb{C}^{d}$ satisfying (1) is called a representing measure of $c^{(n)}$, while the numbers $\int z^{\alpha} \bar{z}^{\beta} d \mu(z)$ are customarily called moments of $\mu$.

Let now $c=\left\{c(\alpha, \beta): \alpha, \beta \in \mathbb{Z}_{+}^{d}\right\}$ be a multisequence of complex numbers. The full (multidimensional and complex) F-moment problem entails determining whether there exists a positive Borel measure $\mu$ on $\mathbb{C}^{d}$ such that supp $\mu \subseteq F$ and

$$
c(\alpha, \beta)=\int z^{\alpha} \bar{z}^{\beta} d \mu(z), \quad \alpha, \beta \in \mathbb{Z}_{+}^{d} .
$$

As above, a positive Borel measure $\mu$ on $\mathbb{C}^{d}$ satisfying (2) is called a representing measure of $\boldsymbol{c}$. We say that a multisequence of moments is determinate if it has precisely one representing measure.

The literature concerning the full $F$-moment problem (not necessarily complex) is extensive and it is still growing (see for instance $[\mathbf{6}, \mathbf{7}, \mathbf{1 8}, \mathbf{1 9}, \mathbf{1}, \mathbf{2 3}, \mathbf{3}, \mathbf{2 2}, \mathbf{4}, \mathbf{2 5}, \mathbf{1 6}$, 30] and $[8,26,27,15,28,5,29]$ where semi-algebraic $F$ 's are considered). The truncated $F$-moment problem has been intensively studied since the early 90 's mostly by Curto

This work was supported at its final stage by the KBN grant \# 2 P03A 00417.

${ }^{1}$ Throughout the whole paper, we tacitly assume that all the functions under the integral sign are absolutely integrable. In particular, by (1), the measure $\mu$ is finite and consequently it is regular (e.g. see [24], Theorem 2.18]). 
and Fialkow (cf. $[\mathbf{2}, \mathbf{2 0}, \mathbf{2 1}, \mathbf{1 1}, \mathbf{1 2}, \mathbf{1 0}, \mathbf{1 3}, \mathbf{1 4}])$. In 1994 R. E. Curto asked a question $^{2}$ whether the truncated $F$-moment problem is more general than the full $F$ moment problem (see also [21, p. 5]). In the same year I answered this question in the affirmative (see [11] for the negative answer to the converse question). The present paper contains the proof of this statement (some ideas involved in it may appear in the literature under different circumstances; for the reader's convenience we include all the details).

An auxiliary result. Denote by $\mathcal{A}^{\prime}$ the dual Banach space of a normed space $\mathcal{A}$ and by $\sigma\left(\mathcal{A}^{\prime}, \mathcal{A}\right)$ the weak-star topology on $\mathcal{A}^{\prime}$. Given a locally compact Hausdorff space $X$, we write $\mathcal{C}_{0}(X)$ for the Banach space (equipped with the supremum norm $\left.\|\cdot\|_{X}\right)$ of all continuous complex functions on $X$ that vanish at infinity. $\mathcal{C}_{\mathrm{c}}(X)$ stands for the set of all $f \in \mathcal{C}_{0}(X)$ such that the closed support of $f$ is compact. The set $\mathcal{C}_{\mathrm{c}}(X)$ is dense in $\mathcal{C}_{0}(X)$ (cf. [24, Theorem 3.17]). We attach to every complex Borel measure $\mu$ on $X$ the functional $\widehat{\mu} \in \mathcal{C}_{0}(X)^{\prime}$ defined by

$$
\widehat{\mu}(f)=\int_{X} f d \mu, \quad f \in \mathcal{C}_{0}(X) .
$$

Proposition 1. Let $F$ be a nonempty closed subset of $\mathbb{C}^{d}$ and let $\rho$ be a nonnegative continuous function on $F$. Assume that $\left\{\mu_{\omega}\right\}_{\omega \in \Omega}$ is a net of finite positive Borel measures on $F$ and $\mu$ is a finite positive Borel measure on $F$ such that

(i) the net $\left\{\widehat{\mu}_{\omega}\right\}_{\omega \in \Omega}$ is $\sigma\left(\mathcal{C}_{\mathrm{c}}(F)^{\prime}, \mathcal{C}_{\mathrm{c}}(F)\right)$-convergent to $\widehat{\mu}$,

(ii) $\sup _{\omega \in \Omega} \int_{F} \rho d \mu_{\omega}<\infty$.

Define the measures $v_{\omega}$ and $v$ on $F$ by $d v_{\omega}=\rho d \mu_{\omega}$ and $d v=\rho d \mu$. Then

(iii) $\nu(F)<\infty$ and the net $\left\{\widehat{v}_{\omega}\right\}_{\omega \in \Omega}$ is $\sigma\left(\mathcal{C}_{0}(F)^{\prime}, \mathcal{C}_{0}(F)\right)$-convergent to $\widehat{v}$.

Moreover, if the set $\{z \in F: \rho(z) \leq r\}$ is compact for some $r>0$, then the net $\left\{\widehat{\mu}_{\omega}\right\}_{\omega \in \Omega}$ is $\sigma\left(\mathcal{C}_{0}(F)^{\prime}, \mathcal{C}_{0}(F)\right)$-convergent to $\widehat{\mu}$ and $\int_{F} f d \mu=\lim _{\omega \in \Omega} \int_{F} f d \mu_{\omega}$ for every $f: F \rightarrow \mathbb{C}$ such that $\frac{f}{1+\rho} \in \mathcal{C}_{0}(F)$.

Proof. Assume that $F$ is not compact. Let $\left\{F_{n}\right\}_{n=1}^{\infty}$ be an increasing sequence of compact subsets of $F$ such that $F=\bigcup_{n=1}^{\infty} F_{n}$. By [24, Theorem 2.12], for every $n \geq 1$ there exists $\psi_{n} \in \mathcal{C}_{\mathrm{c}}(F)$ such that $0 \leq \psi_{n} \leq 1$, and $\psi_{n}=1$ on $F_{n}$. Applying the Lebesgue monotone convergence theorem, (i) and (ii) we obtain

$$
\begin{aligned}
\int_{F} \rho d \mu=\lim _{n \rightarrow \infty} \int_{F n} \rho d \mu & \leq \limsup _{n \rightarrow \infty} \int_{F} \psi_{n} \rho d \mu \\
& =\limsup _{n \rightarrow \infty} \lim _{\omega \in \Omega} \int_{F} \psi_{n} \rho d \mu_{\omega} \leq \limsup _{\omega \in \Omega} \int_{F} \rho d \mu_{\omega}<\infty .
\end{aligned}
$$

According to (ii) and (i), the net $\left\{\widehat{v}_{\omega}\right\}_{\omega \in \Omega} \subseteq \mathcal{C}_{0}(F)^{\prime}$ is uniformly bounded and pointwise convergent on a dense subspace $\mathcal{C}_{\mathrm{c}}(F)$ of $\mathcal{C}_{0}(F)$ to $\widehat{v} \in \mathcal{C}_{0}(F)^{\prime}$. Hence it is $\sigma\left(\mathcal{C}_{0}(F)^{\prime}, \mathcal{C}_{0}(F)\right)$-convergent to $\widehat{v}$ as well ${ }^{3}$.

\footnotetext{
${ }^{2}$ at the Semester on Linear Operators held in the Stefan Banach International Mathematical Center (the organizers: J. Janas, F. H. Szafraniec and J. Zemánek).

${ }^{3}$ Notice that the $\sigma$-compactness of $F$ is not essential in the proof of part (iii) of Proposition 1; indeed, the continuity of $\rho$ and the regularity of $\mu$ (cf. footnote ${ }^{1}$ ) imply the inner regularity of $v$ which, in turn, yields $\nu(F) \leq \sup _{\omega \in \Omega} \int_{F} \rho d \mu_{\omega}<\infty(\operatorname{mimic}(3))$.
} 
Suppose that $K=\{z \in F: \rho(z) \leq r\}$ is compact. Let $\psi \in \mathcal{C}_{\mathrm{c}}(F)$ be such that $0 \leq \psi \leq 1$, and $\psi=1$ on $K$. Since $\lim _{\omega} \int_{F} \psi d \mu_{\omega}=\int_{F} \psi d \mu$, there exists $\omega_{0} \in \Omega$ such that $\int_{F} \psi d \mu_{\omega} \leq M=\int_{F} \psi d \mu+1$ for $\omega \geq \omega_{0}$. This implies that $\mu_{\omega}(K) \leq \int_{F} \psi d \mu_{\omega} \leq M$ for $\omega \geq \omega_{0}$. On the other hand, by (ii), we have $\mu_{\omega}(F \backslash K) \leq \frac{1}{r} \int_{F \backslash K} \rho d \mu_{\omega} \leq$ $\frac{1}{r} \sup _{\tau \in \Omega} \int_{F} \rho d \mu_{\tau}$, for $\omega \in \Omega$, so that $\sup _{\omega \geq \omega_{0}} \mu_{\omega}(F)<\infty$. This means that the net $\left\{\widehat{\mu}_{\omega}\right\}_{\omega \geq \omega_{0}}$ is uniformly bounded and pointwise convergent on $\mathcal{C}_{\mathrm{c}}(F)$ to $\widehat{\mu}$. Consequently, the net $\left\{\widehat{\mu}_{\omega}\right\}_{\omega \in \Omega}$ is $\sigma\left(\mathcal{C}_{0}(F)^{\prime}, \mathcal{C}_{0}(F)\right)$-convergent to $\widehat{\mu}$. Since the net $\left\{\mu_{\omega} \widehat{+} v_{\omega}\right\}_{\omega \in \Omega}$ is $\sigma\left(\mathcal{C}_{0}(F)^{\prime}, \mathcal{C}_{0}(F)\right)$-convergent to $\widehat{\mu+} v$, we get

$$
\int_{F} f d \mu=\int_{F} \frac{f}{1+\rho} d(\mu+v)=\lim _{\omega \in \Omega} \int_{F} \frac{f}{1+\rho} d\left(\mu_{\omega}+v_{\omega}\right)=\lim _{\omega \in \Omega} \int_{F} f d \mu_{\omega}
$$

for every $f: F \rightarrow \mathbb{C}$ such that $\frac{f}{1+\rho} \in \mathcal{C}_{0}(F)$. This completes the proof.

Corollary 2. Let $\{c(m, n): m, n \geq 0, m+n \leq 2 N-1\}$ be a finite sequence of complex numbers $(N \geq 1)$ and let $F$ be a nonempty closed subset of $\mathbb{C}$. Assume that $\left\{\mu_{\omega}\right\}_{\omega \in \Omega}$ is a net of finite positive Borel measures on $F$ and $\mu$ is a finite positive Borel measure on $F$ such that

(i) the net $\left\{\widehat{\mu}_{\omega}\right\}_{\omega \in \Omega}$ is $\sigma\left(\mathcal{C}_{\mathrm{c}}(F)^{\prime}, \mathcal{C}_{\mathrm{c}}(F)\right)$-convergent to $\widehat{\mu}$,

(ii) $c(m, n)=\int_{F} z^{m} \bar{z}^{n} d \mu_{\omega}(z)$ for $m, n \geq 0$ with $m+n \leq 2 N-1$ and $\omega \in \Omega$,

(iii) $\sup _{\omega \in \Omega} \int_{F} z^{N} \bar{z}^{N} d \mu_{\omega}(z)<\infty$.

Then

(iv) $c(m, n)=\int_{F} z^{m} \bar{z}^{n} d \mu(z)$ for $m, n \geq 0$ with $m+n \leq 2 N-1$.

Proof. Apply Proposition 1 to the functions $\rho(z)=z^{N} \bar{z}^{N}$ and $f(z)=z^{m} \bar{z}^{n}(z \in F)$ with $m, n \geq 0$ such that $m+n \leq 2 N-1$ (notice that $\frac{f}{1+\rho} \in \mathcal{C}_{0}(F)$ ).

We emphasize that Corollary 2 is optimum in a sense. Namely, it may happen that the equality in (ii) holds for all $\omega \in \Omega$ and for all integer lattice points $(m, n)$ in the convex triangle $\Delta$ with vertices $(0,0),(0,2 N)$ and $(2 N, 0)$, though no integer lattice point $(m, n)$ belonging to the edge of $\Delta$ joining $(0,2 N)$ and $(2 N, 0)$ satisfies the equality in (iv) (cf. Figure 1). Moreover, the set of all representing measures of a truncated $F$-moment sequence of order $2 N$ may not be $\sigma\left(\mathcal{C}_{0}(F)^{\prime}, \mathcal{C}_{0}(F)\right)$-closed. Example 3 deals with the case $N=1$ and $F=\mathbb{C}$.

EXAmple 3. Since $\sum_{j=1}^{\infty} \frac{1}{j}=\infty$, there exists a strictly increasing sequence of positive integers $\left\{\kappa_{n}\right\}_{n=1}^{\infty}$ such that

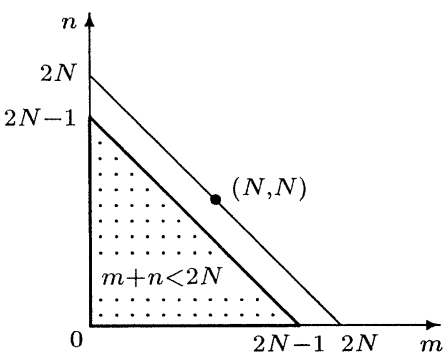

Figure 1. Integer lattice points involved in Corollary 2. 


$$
u_{n} \stackrel{\mathrm{df}}{=} \sum_{j=\kappa_{n}}^{\kappa_{n+1}} \frac{1}{j} \leq \frac{1}{4} \text { for } n \geq 1 \text { and } \lim _{m \rightarrow \infty} u_{m}=\frac{1}{4} \text {. }
$$

Therefore we have

$$
\begin{gathered}
a_{n} \stackrel{\text { df }}{=} \frac{1}{2}+u_{n}-\sum_{j=\kappa_{n}}^{\kappa_{n+1}} \frac{1}{j^{2}}>0, \\
2 b_{n} \stackrel{\text { df }}{=} \frac{1}{2}-u_{n}-\sum_{j=\kappa_{n}}^{\kappa_{n+1}} \frac{1}{j^{3 / 2}}>0
\end{gathered}
$$

and

$$
2 c_{n} \stackrel{\mathrm{df}}{=} \frac{1}{2}-u_{n}+\sum_{j=\kappa_{n}}^{\kappa_{n+1}} \frac{1}{j^{3 / 2}}>0 \text { for } n \geq 1 .
$$

Because $\sum_{j=1}^{\infty} \frac{1}{j^{3 / 2}}<\infty$, (4) yields $\lim _{n \rightarrow \infty} a_{n}=\frac{3}{4}$ and $\lim _{n \rightarrow \infty} b_{n}=\lim _{n \rightarrow \infty} c_{n}=\frac{1}{8}$. Denote by $\delta_{z}$ the probability Borel measure on $\mathbb{C}$ concentrated at the point $z$. Set

$$
\mu \stackrel{\text { df }}{=} \frac{3}{4} \delta_{0}+\frac{1}{8} \delta_{1}+\frac{1}{8} \delta_{-1}
$$

and

$$
\mu_{n} \stackrel{\mathrm{df}}{=} a_{n} \delta_{0}+b_{n} \delta_{1}+c_{n} \delta_{-1}+\sum_{j=\kappa_{n}}^{\kappa_{n+1}} \frac{1}{j^{2}} \delta_{\sqrt{j}} \text { for } n \geq 1
$$

It is a matter of direct verification that $\int f d \mu=\lim _{n \rightarrow \infty} \int f d \mu_{n}$, for every bounded continuous complex function $f$ on $\mathbb{C}$ (in particular $\left\{\widehat{\mu}_{n}\right\}_{n=1}^{\infty}$ is $\sigma\left(\mathcal{C}_{0}(\mathbb{C})^{\prime}, \mathcal{C}_{0}(\mathbb{C})\right.$ )-convergent to $\widehat{\mu})$. Moreover, for every $n \geq 1$, the following conditions hold true

$$
\begin{gathered}
\int z^{0} \bar{z}^{0} d \mu_{n}(z)=\int z^{0} \bar{z}^{0} d \mu(z)=1, \\
\int z^{1} \bar{z}^{0} d \mu_{n}(z)=\int z^{0} \bar{z}^{1} d \mu_{n}(z)=\int z^{1} \bar{z}^{0} d \mu(z)=\int z^{0} \bar{z}^{1} d \mu(z)=0, \\
\int z^{2} \bar{z}^{0} d \mu_{n}(z)=\int z^{1} \bar{z}^{1} d \mu_{n}(z)=\int z^{0} \bar{z}^{2} d \mu_{n}(z)=\frac{1}{2}, \\
\int z^{2} \bar{z}^{0} d \mu(z)=\int z^{1} \bar{z}^{1} d \mu(z)=\int z^{0} \bar{z}^{2} d \mu(z)=\frac{1}{4} .
\end{gathered}
$$

\section{The main result.}

THeOREM 4. Let $F$ be a nonempty closed subset of $\mathbb{C}^{d}$ and let $\{c(\alpha, \beta)\}_{\alpha, \beta \in \mathbb{Z}_{+}^{d}}$ be a multisequence of complex numbers. If for every $n \geq 0$ there exists a positive Borel measure $\mu_{n}$ on F such that

(i) $c(\alpha, \beta)=\int_{F} z^{\alpha} \bar{z}^{\beta} d \mu_{n}(z)$, for all $\alpha, \beta \in \mathbb{Z}_{+}^{d}$ with $|\alpha|+|\beta| \leq n$,

then there exists a positive Borel measure $\mu$ on $F$ such that $c(\alpha, \beta)=\int_{F} z^{\alpha} \bar{z}^{\beta} d \mu(z)$ for all $\alpha, \beta \in \mathbb{Z}_{+}^{d}$. 
Proof. Assume that $F$ is not compact (the other case is simpler). Since $F$ is locally compact metrizable and separable, one can see - applying [9, Theorem V.6.6] to the one-point compactification ${ }^{4}$ of $F-$ that $^{5}$

$$
\mathcal{C}_{0}(F) \text { is a separable Banach space. }
$$

Given $\alpha, \beta \in \mathbb{Z}_{+}^{d}$, we define the function $\varphi_{\alpha, \beta}: \mathbb{C}^{d} \rightarrow \mathbb{C}$ by

$$
\varphi_{\alpha, \beta}(z)=\frac{z^{\alpha} \bar{z}^{\beta}}{\prod_{j=1}^{d}\left(1+\left|z_{j}\right|^{2}\right)^{\alpha_{j}+\beta_{j}+1}}, \quad\left(z \in \mathbb{C}^{d}\right) .
$$

Since the functions $z \mid \rightarrow \frac{z^{m} \bar{z}^{n}}{\left(1+|z|^{2}\right)^{m+n+1}}(m, n \geq 0)$ are in $\mathcal{C}_{0}(\mathbb{C})$ and the $d$-fold tensor product of $\mathcal{C}_{0}$ functions is again a $\mathcal{C}_{0}$ function, we conclude that

$$
\varphi_{\alpha, \beta} \in \mathcal{C}_{0}(F), \quad \alpha, \beta \in \mathbb{Z}_{+}^{d} .
$$

It follows from (i) that $\left|\widehat{\mu}_{n}(f)\right| \leq \int_{F} z^{0} \bar{z}^{0} d \mu_{n}(z)\|f\|_{F}=c(0,0)\|f\|_{F}$, for every $f \in \mathcal{C}_{0}(F)$ and so $\widehat{\mu}_{n}$ belongs to $c(0,0) \boldsymbol{B}$, where $\boldsymbol{B}$ is the closed unit ball in $\mathcal{C}_{0}(F)^{\prime}$. By (5), the set $c(0,0) \boldsymbol{B}$ is weak-star metrizable and weak-star compact (cf. [9, Theorems V.3.1 and V.5.1]). Hence there exists a subsequence $\left\{\widehat{\mu}_{k_{n}}\right\}_{n=0}^{\infty}$ of $\left\{\widehat{\mu}_{n}\right\}_{n=0}^{\infty}$ that is weakstar convergent to a functional $\Lambda \in c(0,0) \boldsymbol{B}$. Notice that if $f \in \mathcal{C}_{0}(F)$ and $f \geq 0$, then $\Lambda(f)=\lim _{n \rightarrow \infty} \widehat{\mu}_{k_{n}}(f) \geq 0$ and so by the Riesz representation theorem (cf. [24, Theorems 2.14 and 6.19$]$ or $[\mathbf{1 7}, \S 56]$ ) there exists a finite positive Borel measure $\mu$ on $F$ such that $\Lambda=\widehat{\mu}$. If $n(\alpha) \in \mathbb{Z}_{+}$is chosen so that $k_{n(\alpha)} \geq 2|\alpha|$, then, by (i), we have

$$
\int_{F} z^{\alpha} \bar{z}^{\alpha} d \mu_{k_{n}}(z)=c(\alpha, \alpha) \text { for } n \geq n(\alpha)\left(\alpha \in \mathbb{Z}_{+}^{d}\right) .
$$

Applying Proposition 1 to $\rho(z)=z^{\alpha} \bar{z}^{\alpha}$ gives us $\int_{F} z^{\alpha} \bar{z}^{\alpha} d \mu(z)<\infty$ for $\alpha \in \mathbb{Z}_{+}^{d}$ and

$$
\lim _{n \rightarrow \infty} \int_{F} f(z) z^{\alpha} \bar{z}^{\alpha} d \mu_{k_{n}}(z)=\int_{F} f(z) z^{\alpha} \bar{z}^{\alpha} d \mu(z), \quad f \in \mathcal{C}_{0}(F), \alpha \in \mathbb{Z}_{+}^{d} .
$$

It follows from (i), (6) and (7) that

$$
\begin{aligned}
c(\alpha, \beta) & =\lim _{n \rightarrow \infty} \int_{F} z^{\alpha} \bar{z}^{\beta} d \mu_{k_{n}}(z) \\
& =\lim _{n \rightarrow \infty} \int_{F} \varphi_{\alpha, \beta}(z) \prod_{j=1}^{d}\left(1+\left|z_{j}\right|^{2}\right)^{\alpha_{j}+\beta_{j}+1} d \mu_{k_{n}}(z) \\
& =\int_{F} \varphi_{\alpha, \beta}(z) \prod_{j=1}^{d}\left(1+\left|z_{j}\right|^{2}\right)^{\alpha_{j}+\beta_{j}+1} d \mu(z) \\
& =\int_{F} z^{\alpha} \bar{z}^{\beta} d \mu(z), \quad \alpha, \beta \in \mathbb{Z}_{+}^{d},
\end{aligned}
$$

which completes the proof.

\footnotetext{
${ }^{4}$ One can show that if $X$ is a locally compact Hausdorff space that is not compact, then the one-point compactification of $X$ is metrizable if and only if $X$ is metrizable and separable.

${ }^{5}$ The separability of $\mathcal{C}_{0}(F)$ can also be deduced from the Stone-Weierstrass theorem.
} 
REMARK 5. In fact, we have proved that if for every $n \geq 0$ there exists a positive Borel measure $\mu_{n}$ on $F$ satisfying condition (i) of Theorem 4 , then there exists a positive Borel measure $\mu$ on $F$ with all its moments finite and a subsequence $\left\{\mu_{k_{n}}\right\}_{n=0}^{\infty}$ of $\left\{\mu_{n}\right\}_{n=0}^{\infty}$ such that $\left\{\widehat{\mu_{k_{n}}^{\alpha}}\right\}_{n=0}^{\infty}$ is $\sigma\left(\mathcal{C}_{0}(F)^{\prime}, \mathcal{C}_{0}(F)\right)$-convergent to $\widehat{\mu^{\alpha}}$ for $\alpha \in \mathbb{Z}_{+}^{d}$; here $d \nu^{\alpha}(z)=z^{\alpha} \bar{z}^{\alpha} d \nu(z)$ for $v=\mu, \mu_{n}$. This, in turn, has enabled us to show that $\mu$ is a representing measure of $\{c(\alpha, \beta)\}_{\alpha, \beta \in \mathbb{Z}_{+}^{d}}$. It is clear that all representing measures of $\{c(\alpha, \beta)\}_{\alpha, \beta \in \mathbb{Z}_{+}^{d}}$ can be obtained by way of this limit procedure. In case $\mu$ is unique we can prove more.

THEOREM 6. Let $\{c(\alpha, \beta)\}_{\alpha, \beta \in \mathbb{Z}_{+}^{d}}, F, \mu_{n}$ and $\mu$ be as in Theorem 4. If, moreover, the multisequence $\{c(\alpha, \beta)\}_{\alpha, \beta \in \mathbb{Z}_{+}^{d}} \widehat{i s}^{+}$determinate, then the sequence $\left\{\widehat{\mu_{n}^{\alpha}}\right\}_{n=0}^{\infty}$ is $\sigma\left(\mathcal{C}_{0}(F)^{\prime}, \mathcal{C}_{0}(F)\right)$-convergent to $\widehat{\mu^{\alpha}}$, for every $\alpha \in \mathbb{Z}_{+}^{d}$.

Proof. Analysis similar to that in the proof of Theorem 4 (cf. Remark 5) shows that for every subsequence $\left\{\mu_{k_{n}}\right\}_{n=0}^{\infty}$ of $\left\{\mu_{n}\right\}_{n=0}^{\infty}$ there exists a subsequence $\left\{\mu_{k_{n}}\right\}_{n=0}^{\infty}$ of $\left\{\mu_{k_{n}}\right\}_{n=0}^{\infty}$ such that $\left\{\widehat{\mu_{k_{l n}}^{\alpha}}\right\}_{n=0}^{\infty}$ is $\sigma\left(\mathcal{C}_{0}(F)^{\prime}, \mathcal{C}_{0}(F)\right)$-convergent to $\widehat{\mu^{\alpha}}$ for $\alpha \in \mathbb{Z}_{+}^{d}$ (use the fact that the representing measure $\mu$ is unique). Hence the general topological characterization of convergent sequences yields the conclusion.

It is worth while to notice that if the multisequence of moments $\{c(\alpha, \beta)\}_{\alpha, \beta \in \mathbb{Z}_{+}^{d}}$ is not determinate, then $\left\{\widehat{\mu}_{n}\right\}_{n=0}^{\infty}$ may not be $\sigma\left(\mathcal{C}_{0}(F)^{\prime}, \mathcal{C}_{0}(F)\right)$-convergent. Indeed, if $\mu \neq v$ are two representing measures of $\{c(\alpha, \beta)\}_{\alpha, \beta \in \mathbb{Z}_{+}^{d}}$ and the sequence $\left\{\mu_{n}\right\}_{n=0}^{\infty}$ is defined by $\mu_{2 k}=\mu$ and $\mu_{2 k+1}=v$ for $k \geq 0$, then $\left\{\mu_{n}^{+}\right\}_{n=0}^{\infty}$ satisfies condition (i) of Theorem 4 but $\left\{\widehat{\mu}_{n}\right\}_{n=0}^{\infty}$ is not $\sigma\left(\mathcal{C}_{0}(F)^{\prime}, \mathcal{C}_{0}(F)\right)$-convergent (indeed, otherwise it must be $\widehat{\mu}=\widehat{v}$ which, by the Riesz representation theorem (see also footnote ${ }^{1}$ ), gives us $\mu=v$, a contradiction).

Theorems 4 and 6 can easily be adapted to the context of other classical moment problems and in particular to the multidimensional Hamburger moment problem.

Acknowledgements. The author would like to thank Professor R. E. Curto for his encouragement to write this paper.

\section{REFERENCES}

1. N. I. Akhiezer, The classical moment problem (Hafner Publ. Co., New York, 1965).

2. T. Ando, Truncated moment problems for operators, Acta Sci. Math. (Szeged) 31 (1970), 319-333.

3. A. Atzmon, A moment problem for positive measures on the unit disc, Pacific $J$. Math. 59 (1975), 317-325.

4. C. Berg, J. P. R. Christensen and C. U. Jensen, A remark on the multidimensional moment problem, Math. Ann. 243 (1979), 163-169. $397-429$.

5. T. M. Bisgaard, Extensions of Hamburger's theorem, Semigroup Forum 57 (1998), 1682.

6. T. Carleman, Sur le problème des moments, C. R. Acad. Sci. Paris 174 (1922), 1680-

7. T. Carleman, Sur les équations intégrales singuliéres à noyau réel et symétrique (Uppsala Universitets Arsskrift, Uppsala, 1923).

8. G. Cassier, Problème des moments sur un compact de $\mathbb{R}^{n}$ et décomposition de polynómes à plusieurs variables, J. Funct. Anal. 58 (1984), 254-266.

9. J. B. Conway, A course in functional analysis (Springer-Verlag, 1985). 
10. R. E. Curto, An operator-theoretic approach to truncated moment problems, in Linear operators, J. Janas, F. H. Szafraniec and J. Zemánek (eds.) (Banach Center Publications 38, Warszawa, 1997), 75-104.

11. R. E. Curto and L. A. Fialkow, Recursiveness, positivity, and truncated moment problems, Houston J. Math. 17 (1991), 603-635.

12. R. E. Curto and L. A. Fialkow, Solution of the truncated complex moment problem for flat data, Mem. Amer. Math. Soc. 119 (1996).

13. R. E. Curto and L. A. Fialkow, Flat extensions of positive moment matrices: Recursively generated relations, Mem. Amer. Math. Soc., to appear. 1998.

14. R. E. Curto and L. A. Fialkow, The truncated complex $K$-moment problem, preprint

15. R. E. Curto and M. Putinar, Nearly subnormal operators and moment problems, $J$. Funct. Anal. 115 (1993), 480-497.

16. B. Fuglede, The multidimensional moment problem, Expo. Math. 1 (1983), 47-65.

17. P. R. Halmos, Measure theory (Springer-Verlag, 1974).

18. E. K. Haviland, On the momentum problem for distributions in more than one dimension, Amer. J. Math. 57 (1935), 562-568.

19. E. K. Haviland, On the momentum problem for distributions in more than one dimension, II, Amer. J. Math. 58 (1936), 164-168.

20. M. G. Krein and A. Nudel'man, The Markov moment problem and extremal problems, Transl. Math. Monographs 50 (Amer. Math. Soc., Providence, RI, 1977).

21. H. Landau, Classical background of the moment problem, in Moments in Mathematics, Proc. Sympos. Appl. Math. 37 (1987), 1-15.

22. P. H. Maserick, Moments of measures on convex bodies, Pacific J. Math. 68 (1977), $135-152$.

23. A. E. Nussbaum, Quasi-analytic vectors, Ark. Mat. 6 (1967), 179-191.

24. W. Rudin, Real and complex analysis (McGraw-Hill, 1974).

25. K. Schmüdgen, An example of a positive polynomial which is not a sum of squares of polynomials. A positive, but not strongly positive functional, Math. Nachr. 88 (1979), 385390.

26. K. Schmüdgen, The $K$-moment problem for compact semi-algebraic sets, Math. Ann. 289 (1991), 203-206.

27. J. Stochel, Moment functions on real algebraic sets, Ark. Mat. 30 (1992), 133-148.

28. J. Stochel and F. H. Szafraniec, Algebraic operators and moments on algebraic sets, Portugal. Math. 51 (1994), 25-45.

29. J. Stochel and F. H. Szafraniec, The complex moment problem and subnormality: a polar decomposition approach, J. Funct. Anal. 159 (1998), 432-491.

30. F. H. Szafraniec, Moments on compact sets, in Prediction theory and harmonic analysis, V. Mandrekar and H. Salehi (eds.) (North-Holland, Amsterdam, 1983), 379-385. 